\title{
Erratum to: Multivariate study of predictive factors for clearly defined lung lesions without visible endobronchial lesions in transbronchial biopsy
}

\author{
Maribel Botana-Rial · Manuel Núñez-Delgado • Abel Pallarés-Sanmartín • \\ Virginia Leiro-Fernández • María Torres-Durán • \\ Cristina Represas-Represas • Alberto Fernández-Villar
}

Published online: 22 July 2010

(C) Springer Science+Business Media, LLC 2010

\section{Erratum to: Surg Endosc}

\section{DOI 10.1007/s00464-010-1080-4}

The correct spelling of all authors is: Maribel Botana-Rial, Manuel Núñez-Delgado, Abel Pallarés-Sanmartín, Virginia Leiro-Fernández, María Torres-Durán, Cristina RepresasRepresas, Alberto Fernández-Villar.

\footnotetext{
The online version of the original article can be found under doi:10.1007/s00464-010-1080-4.

M. Botana-Rial · M. Núñez-Delgado · A. Pallarés-Sanmartín ·

V. Leiro-Fernández · M. Torres-Durán ·

C. Represas-Represas · A. Fernández-Villar ( $₫)$

Unit of Interventional Bronchopleural Pathology,

Pneumology Department, University Hospital Complex

of Vigo (CHUVI), Pizarro 22, 36204 Vigo, Pontevedra,

Spain

e-mail: alberto.fernandez.villar@sergas.es

M. Botana-Rial

e-mail: maria.isabel.botana.rial@sergas.es
} 